\title{
Chronic rhinosinusitis severity is associated with need for asthma-related systemic corticosteroids*
}

\author{
Katie M. Phillips ${ }^{1,2}$, Lloyd P. Hoehle1,2, Regan W. Bergmark',2, Adam P. \\ Campbell1,2, David S. Caradonna 1,3, Stacey T. Gray ${ }^{1,2}$, Ahmad R. Sedaghat 1,2,3,4 \\ Rhinology 55: 211-217, 2017 \\ https://doi.org/10.4193/Rhino17.029 \\ Department of Otolaryngology, Harvard Medical School, Boston, MA, USA \\ *Received for publication: \\ Department of Otolaryngology, Massachusetts Eye and Ear Infirmary, Boston, MA, USA \\ February 10, 2017 \\ Division of Otolaryngology, Beth Israel Deaconess Medical Center, Boston, MA, USA \\ Accepted: April 10, 2017 \\ ${ }^{4}$ Department of Otolaryngology and Communications Enhancement, Boston Children's Hospital, Boston, MA, USA
}

\begin{abstract}
Background: Chronic rhinosinusitis (CRS) is highly prevalent in patients with asthma. However, no study has evaluated the effect of CRS severity on asthma-related oral corticosteroid use - a marker of poor asthma control and prognosis. We therefore sought to evaluate the association between CRS severity and asthma-related oral corticosteroid use.
\end{abstract}

Methodology: Prospective cross-sectional study of 110 adult asthmatic CRS patients. CRS severity was measured using the 22item Sinonasal Outcomes Test (SNOT-22) and Lund-Kennedy endoscopy score. Number of asthma-related courses of oral corticosteroids in the past year was queried at enrollment. Association was sought between metrics for CRS severity and asthma-related oral corticosteroids use in the last year. Receiver operating characteristic (ROC) curves defined whether SNOT-22 or endoscopy scores could be used for detecting asthma-related oral corticosteroid use.

Results: The mean SNOT-22 score was 44.9 (standard deviation [SD] : 23.3) and mean endoscopy score was 4.1 (SD: 3.0). The mean number of asthma-related oral corticosteroid courses taken in the last year was 1.1 (SD: 1.9). SNOT-22, but not endoscopy score, was associated with requiring at least one course of asthma-related oral corticosteroids in the last year (odds ratio $=1.03,95 \% \mathrm{Cl}$ : $1.02-1.06, p=0.003$ ), which translates to an odds ratio of 2.0 for a 21-point increase in SNOT-22. ROC analysis identified equally optimal SNOT-22 scores of greater than 32 (sensitivity: $88.1 \%$, specificity: $41.2 \%$ ) or greater than 65 (sensitivity: $38.1 \%$, specificity: 91.2\%) for detecting the need for at least one course of oral corticosteroids within the past year.

Conclusions: CRS symptom severity is associated with past asthma-related oral corticosteroid use. SNOT-22 scores may be used as a versatile tool to screen for past asthma-related oral corticosteroid use in asthmatic CRS patients - i.e. those at greatest risk from their asthma - with either high sensitivity or high specificity.

Key words: asthma, chronic rhinosinusitis, corticosteroids, severity, SNOT-22, endoscopy score

\section{Introduction}

Chronic rhinosinusitis (CRS) is an inflammatory disease of the sinonasal mucosa that impacts patient quality of life (QOL) through sinonasal symptomatology, acute CRS exacerbations and exacerbation of comorbid pulmonary diseases ${ }^{(1-3)}$. CRS is a multifactorial disease and may arise and persist due to many different pathophysiologic mechanisms ${ }^{(4-8)}$. From the pathophysiologic standpoint, CRS and asthma are diseases which are both characterized by inflammation of the airway - affecting the upper and lower airway, respectively ${ }^{(9-11)}$. These two diseases are known to be epidemiologically associated with each other and there is evidence that one disease may be related to the development of the other ${ }^{(12-17)}$. Up to one half of patients with CRS and nasal polyps have comorbid asthma ${ }^{(18)}$. Similarly, the prevalence of comorbid CRS in known asthmatics has been reported to be as high as $40 \%{ }^{(19)}$.

In order to optimize the management of CRS patients, it is important to understand how one disease process influences the other. It could be particularly helpful to know how metrics 
of CRS may inform clinical caretakers about their CRS patients' asthma status. CRS severity, as measured by radiographic findings, is strongly associated with the diagnosis of asthma,(20) and greater radiographic severity of CRS is associated with increasing asthma severity ${ }^{(21)}$. Comorbid CRS is associated with increased frequency of asthma exacerbations (22), and increasing sinonasal symptom severity is associated with decreased asthma control in asthmatics with CRS (3). It is unknown, however, how the degree of CRS symptom severity relates to the use of systemic corticosteroids for asthma. This is a particularly important relationship to understand because past systemic corticosteroid use has a great deal of clinical significance as an asthma outcome measure. The past need for asthma-related systemic corticosteroids is a reflection of asthma severity, the frequency of asthma exacerbations, poor pulmonary function, asthma-related emergency department visits and asthma-related mortality ${ }^{(11,23,24)}$. Therefore, asthma-related systemic corticosteroid use is an important outcome measure for asthma because it not only captures global asthma characteristics, such as disease severity, but it also captures past asthma manifestations, current asthma characteristics and future asthma-related prognosis as well.

Given the clinical importance of systemic corticosteroid use in asthmatics, understanding how CRS may impact this important asthma-related outcome measure may be clinically informative by helping to identify the asthmatic CRS patients who might be most at risk from their asthma. In this study, we sought to characterize how the severity of CRS relates to the need for asthma-related oral corticosteroids. Our hypothesis was that patients with greater CRS severity, as reflected by severity of sinonasal symptoms and/or endoscopic findings, would have required a greater number of asthma-related oral corticosteroids in the past.

\section{Materials and methods}

Study participants

This study was approved by the Massachusetts Eye and Ear Infirmary Human Studies Committee and meets the ethical principles of the Declaration of Helsinki. A total of 110 participants (age 18 or older) with CRS and asthma were recruited consecutively, prospectively and provided informed consent for inclusion in this study. All participants met consensus guidelines established for CRS (9). All patients were referred to us with a previous diagnosis of asthma based on previously published guidelines (11), which was also confirmed based on a previous clinical history and symptomatology ${ }^{(11)}$. Participants were not excluded based on asthma severity and therefore our study cohort represented patients with all levels of asthma severity. Exclusion criteria included comorbid diagnosis of vasculitis, cystic fibrosis, sarcoidosis and immunodeficiency. Individuals in the midst of an acute CRS exacerbation were also excluded.

\section{Study design and data collection}

This is a prospectively designed cross-sectional study to investigate the association of CRS severity on the need for asthmarelated oral corticosteroid use over the past year. All data was collected at enrollment. Demographic information was collected including age, gender. Data collected directly from all participants included CRS-related symptomatology as measured by the validated 22-item Sinonasal Outcomes Test (SNOT-22) survey (25). Since individuals having an acute CRS exacerbation were excluded, we took the SNOT-22 score of our study participants to be reflective of their baseline CRS symptom severity. The number of oral corticosteroid courses used in the past year for asthma (an outcome measure of asthma used by the National Heart, Lung, and Blood Institute Expert Panel Report 3 on the Guidelines for the Diagnosis and Management of Asthma) (11), use of a corticosteroid-containing inhaler for asthma, intranasal steroid use and history of previous sinus surgery. Tobacco use was assessed by asking participants if they were current or former smokers. For each participant, the evaluating rhinologist assessed 1) aeroallergen hypersensitivity based on formal skin or serological allergy testing, 2) a history of aspirin sensitivity, and 3) the presence of nasal polyps. A Lund-Kennedy endoscopy score was calculated from the evaluating rhinologist's nasal endoscopy ${ }^{(26)}$.

\section{Statistical analysis}

All analysis was performed using the statistical software package $\mathrm{R}$ (www.r-project.org). A total of 110 patients were recruited in order to have at least $80 \%$ power to detect an association of medium effect size (Cohen's $d=0.15$ ) between our metrics of CRS severity (SNOT-22 and endoscopy scores) and asthma-related oral corticosteroid use at a significance level of 0.05 , while controlling for eight confounding variables. All correlations were performed with Pearson's correlation. Univariate and multivariable associations with the number of oral corticosteroid courses used in the past year for asthma as the dependent variable were calculated using negative binomial regression. Univariate and multivariable associations with any asthma-related oral corticosteroids used in the past year (as the dependent variable) were performed using logistic regressions. Analysis of Receiver Operating Characteristic (ROC) curves was performed with the pROC package ${ }^{(27)}$. The area under the ROC curve (AUC) was calculated with the trapezoid rule using the auc() function and the $95 \%$ confidence interval of the AUC was calculated by performing 2000 bootstraps of the data with the ci() function. P-value for significance of the ROC curve was determined by Wilcoxon rank-sum test.

\section{Results}

Characteristics of study participants

A total of 110 participants, $37.3 \%$ males and $62.7 \%$ females, with 
Table 1. Characteristics of study participants

\begin{tabular}{|lc|}
\hline Demographics & $\begin{array}{c}\text { Study participants } \\
(\mathbf{N}=\mathbf{1 1 0})\end{array}$ \\
\hline Age, mean in years, (SD) & $48.8(15.4)$ \\
\hline Gender & \\
\hline Male & $37.3 \%$ \\
\hline Female & $62.7 \%$ \\
\hline Smoking & $32.7 \%$ \\
\hline Comorbidities & \\
\hline Aeroallergen hypersensitivity & $66.4 \%$ \\
\hline Aspirin sensitivity & $10.9 \%$ \\
\hline CRS characteristics & \\
\hline Nasal polyps & $60.0 \%$ \\
\hline Previous sinus surgery & $50.9 \%$ \\
\hline Intranasal steroid use & $82.7 \%$ \\
\hline SNOT-22 score, mean (SD) & $44.9(23.3)$ \\
\hline Endoscopy score, mean (SD) & $4.1(3.0)$ \\
\hline Asthma characteristics & \\
\hline Asthma controller inhaler & $40.9 \%$ \\
\hline Oral corticosteroids in last year, mean (SD) & $1.1(1.9)$ \\
\hline
\end{tabular}

CRS and asthma were recruited with a mean age of 48.8 years (SD: 15.4). The characteristics of the study participants are summarized in Table 1. Of these participants, $32.7 \%$ were current or former cigarette smokers, $66.4 \%$ had at least one aeroallergen hypersensitivity, $10.9 \%$ had aspirin sensitivity, $60.0 \%$ had nasal polyps, $50.9 \%$ had a history of previous sinus surgery, $82.7 \%$ reported intranasal steroid use. Because we did not exclude patients based on asthma severity or asthma-related control medication usage, only $40.9 \%$ reported consistent asthma controller inhaler use. The mean SNOT-22 score was 44.9 (SD: 23.3, range: $0-100$ ) and mean endoscopy score was 4.1 (SD: 3.0, range: $0-12$ ). The mean number of asthma-related oral corticosteroid courses used in the past year was 1.1 (SD: 1.9), with $38.2 \%$ of the participants having used at least one course of asthma-related oral corticosteroids in the last year.

CRS severity is associated with past asthma-related oral corticosteroid use

In this study, we used SNOT-22 score and nasal endoscopy score as measures of CRS severity and found that SNOT-22 and nasal endoscopy score were correlated $(r=0.22, p=0.022)$. We evaluated the relationship between SNOT-22 and endoscopy score, as measures of CRS severity, and the number of asthma-related oral corticosteroids courses taken in the past year (Figure 1). We found a statistically significant positive correlation between the number of asthma-related oral corticosteroids courses in the past year and SNOT-22 $(r=0.37, p<0.001)$, with the correlation
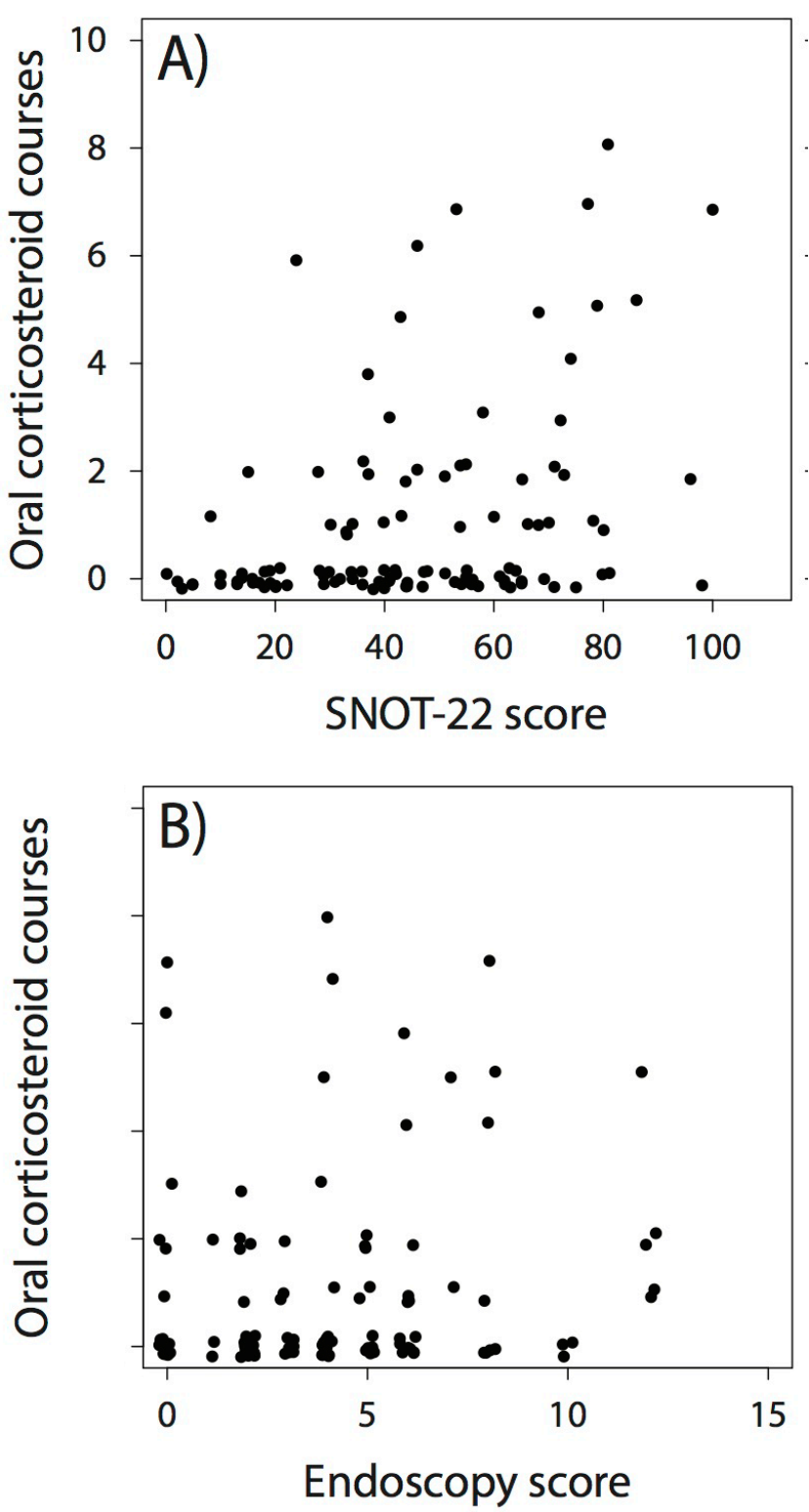

Figure 1. Scatter plot of the number of asthma-related oral corticosteroid courses taken in the last year vs. (A) SNOT-22 score and (B) endoscopy score.

coefficient reflecting a correlation of medium effect size. We did not find a significant correlation between the number of asthma-related oral corticosteroids courses in the past year and endoscopy score $(r=0.14, p=0.157)$ with the point estimate of the correlation coefficient suggesting that if a correlation does exist between these two variables, it would be of small effect size. We then sought formal associations between CRS symptom severity, as reflected by the SNOT-22 score, and endoscopy score and the number of asthma-related oral corticosteroid courses taken in the last year. SNOT-22 score was associated with number of asthma related oral corticosteroid courses taken in the last year on univariate analysis (relative risk $[\mathrm{RR}]=1.03,95 \% \mathrm{Cl}$ : 1.01 - 
$1.04, \mathrm{p}<0.001)$. This association remained statistically significant $(\mathrm{RR}=1.03,95 \% \mathrm{Cl}: 1.02-1.05, \mathrm{p}<0.001)$ after controlling for age, gender, aeroallergen sensitivity, nasal polyps, endoscopy score, intranasal corticosteroid use, smoking status, and the use of an asthma controller inhaler. This result indicated that the risk for asthma-related oral corticosteroid use increased 2 fold for every 22-point increase in the SNOT-22 score. In contrast, endoscopy score was not associated with the number of asthma-related oral corticosteroid courses in the last year ( $\mathrm{RR}=1.07,95 \% \mathrm{Cl}$ : 0.95 $-1.20, p=0.276$ ).

Next we evaluated the associations between SNOT-22 and endoscopy scores with requiring at least one course of asthmarelated oral corticosteroids in the past year. SNOT-22 score was associated with asthma-related oral corticosteroid use in the past year on univariate analysis (odds ratio $[\mathrm{OR}]=1.03,95 \% \mathrm{Cl}$ : $1.01-1.05, p=0.001$ ). Controlling for the same covariates as listed above, this association remained statistically significant $(\mathrm{OR}=1.03,95 \% \mathrm{Cl}: 1.01-1.06, \mathrm{p}=0.003)$. This result corresponds to an $\mathrm{OR}=2$ for every 21-point increase in SNOT-22. Endoscopy score was also associated with having used at least one asthmarelated oral corticosteroid course in the last year $(\mathrm{OR}=1.16$, $95 \% \mathrm{Cl}: 1.01-1.32, \mathrm{p}=0.031$ ) on univariate analysis. However, after controlling for age, gender, aeroallergen sensitivity, nasal polyps, SNOT-22 score, intranasal corticosteroid use, smoking status, and the use of an asthma controller inhaler, endoscopy score was no longer significantly associated with any asthmarelated oral corticosteroid use in the past year $(\mathrm{OR}=1.12,95 \% \mathrm{Cl}$ : $0.95-1.33, p=0.181)$.

Finally, because asthma may directly contribute to certain symptoms represented on the SNOT-22, we sought to better understand whether the associations we found between the SNOT22 score and past asthma-related oral corticosteroid usage were related to SNOT-22 items that could potentially be related to the diagnosis of asthma rather than CRS. We chose the severity of "cough" as the SNOT-22 item that could potentially have the greatest contribution from asthma. We also chose the severity of "blockage/congestion of nose" as a SNOT-22 item that is likely to have an insignificant contribution from comorbid asthma. After controlling for the same covariates described above, the severity of "cough" was associated with the number of asthmarelated oral corticosteroid courses $(\mathrm{RR}=1.30,95 \% \mathrm{Cl}$ : $1.06-1.59$, $\mathrm{p}=0.013)$ and having taken at least one course of asthma-related oral corticosteroids in the last year $(\mathrm{OR}=1.62,95 \% \mathrm{Cl}: 1.18-2.23$, $\mathrm{p}=0.003)$. Similarly, after controlling for the same covariates, the severity of "blockage/congestion of nose" was associated with the number of asthma-related oral corticosteroid courses (RR $=1.58,95 \% \mathrm{Cl}: 1.25-2.00, \mathrm{p}<0.001)$ and having taken at least one course of asthma-related oral corticosteroids in the last year (adjusted $\mathrm{OR}=1.58,95 \% \mathrm{Cl}: 1.12-2.22, \mathrm{p}=0.009$ ). We found
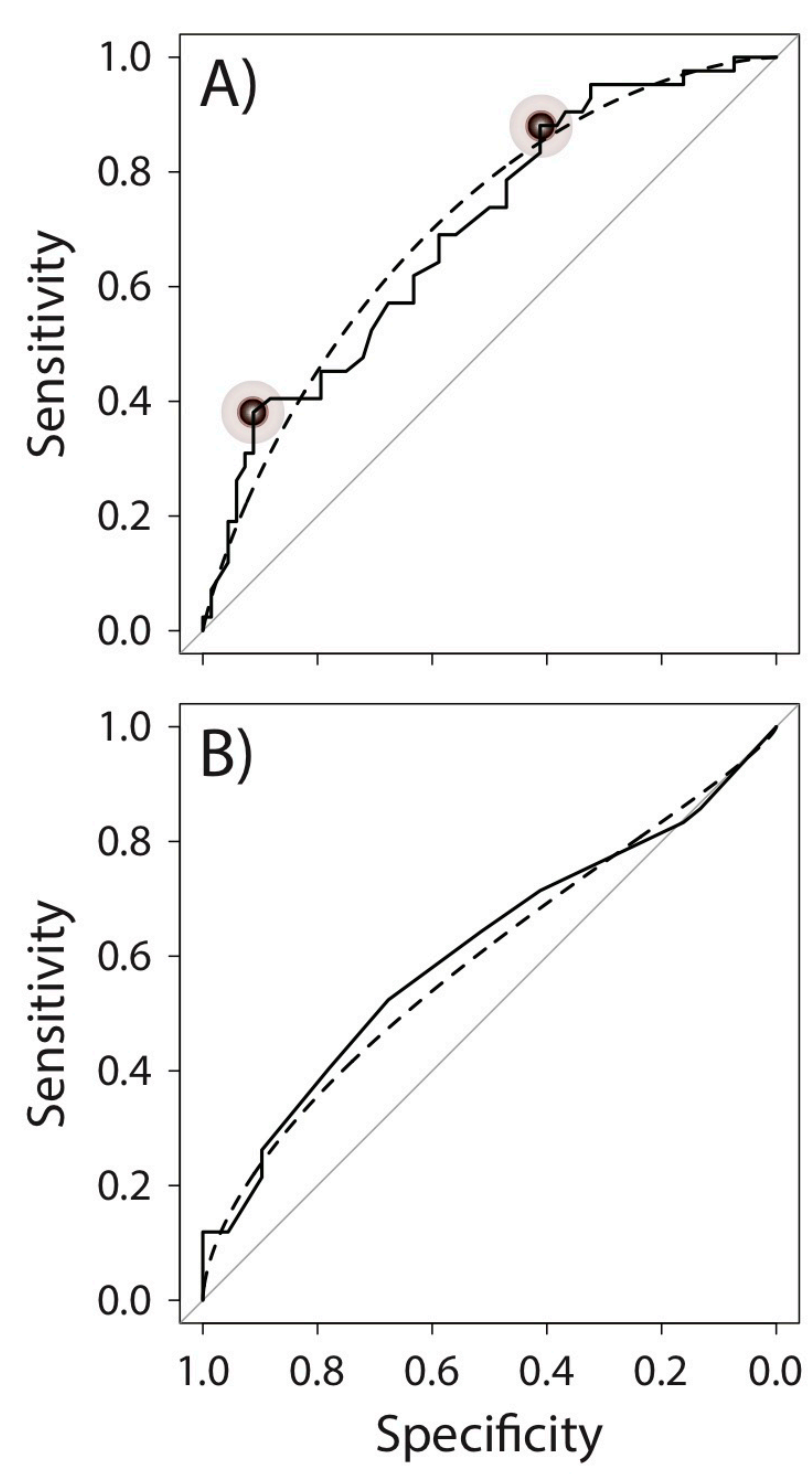

Figure 2. ROC curve for the use of (A) SNOT-22 and (B) endoscopy score to detect those asthmatic CRS patients who have required oral corticosteroids in the last year for asthma-related indications. The points reflecting a SNOT-22 score cut-off of greater than 32 and greater than 65 , which equally maximized the sum of sensitivity and specificity, are marked on panel $(A)$.

qualitatively similar associations for other nasal symptoms (unlikely to be due to asthma) and sleep symptoms (also potentially contributed to by asthma) on the SNOT-22. That all of these symptoms similarly associate with asthma-related oral corticosteroid use in the past year suggests that these associationsand the association we find with the total SNOT-22 score-are predominantly due to the same process, CRS.

SNOT-22 score as a tool for detecting asthma-related oral corticosteroid usage in the past year In order to determine whether SNOT-22 or endoscopy scores 
could be used to detect participants needing at least one course of oral corticosteroids for asthma exacerbations, we generated ROC curves for these two metrics of CRS severity (Figure 2). The area-under-the-curve (AUC) for the SNOT-22 ROC curve was significantly greater than 0.5 ( $A \cup C=0.70,95 \% \mathrm{Cl}: 0.60-$ $0.80, p=0.001$ ) indicating that SNOT-22 score may be useful for detecting asthma-related oral corticosteroid use in asthmatic CRS patients. We calculated that SNOT-22 score thresholds of greater than 32 and greater than 65 equally maximized the sum of sensitivity and specificity for detecting asthma-related oral corticosteroid use in the past year. Using a SNOT-22 score threshold of greater than 32 provided $88.1 \%$ sensitivity and $41.2 \%$ specificity for detecting the need for at least one course of asthma-related oral corticosteroids in the last year for asthmatic CRS patients. Alternatively, a SNOT-22 score threshold of greater than 65 provided $38.1 \%$ sensitivity and $91.2 \%$ specificity for detecting the need for at least one course of asthma-related oral corticosteroids in the last year. The ROC curve for endoscopy score, however, did not have an AUC significantly greater than 0.5 ( $A U C=0.61,95 \% \mathrm{Cl}: 0.49-0.72, \mathrm{p}=0.057$ ), indicating that endoscopy score was not useful in our cohort for detecting asthma-related oral corticosteroids in the last year.

\section{Discussion}

Asthma and CRS are commonly comorbid illnesses that, in combination, have a negative impact on the clinical outcomes of both conditions ${ }^{(28-31)}$. Asthma is associated with worse CRS symptomatology and CRS is associated with worse asthma outcomes. For example, asthmatics with CRS are at increased risk for acute asthma exacerbations, which not only cause a decrease in QOL and have a substantial economic impact for patients ${ }^{(32)}$, but they can also result in severe morbidity and death ${ }^{(11)}$. One measure of asthma exacerbations, and an indicator of the severity of the exacerbation, is the need for systemic corticosteroids (11). Therefore, a greater understanding of the association between CRS severity and asthma-related systemic corticosteroid use could provide further insight into how CRS outcome measures can be used to monitor comorbid asthma status. In this study, we sought to determine whether the severity of CRS was associated with past utilization of asthma-related oral corticosteroids in asthmatic CRS patients. We found a significant, positive association between the severity of CRS symptomatology and the need for asthma-related oral corticosteroid use. Additionally, we found that the SNOT-22 score may be used with either a high sensitivity or high specificity to detect asthmatic CRS patients who may have needed oral corticosteroids for asthma exacerbations in the past year.

Comorbid CRS is associated with worse asthma outcome measures. CRS, as a comorbid illness to asthma, is associated with increased frequency of asthma exacerbations ${ }^{(23)}$ and asthma severity ${ }^{(33)}$. Increased radiologic severity of CRS is associated with increased severity of asthma ${ }^{(21,30)}$. Additionally, comorbid CRS is associated with decreased QOL and poorer lung function in asthmatics ${ }^{(29)}$. However, little is known about the association between the severity of CRS and asthma outcome measures. The current study investigates whether CRS severity is related to the need for asthma-related oral corticosteroids, a measure of asthma exacerbations, and a risk factor for asthma-related health care cost and mortality ${ }^{(11,32)}$.

In this study, we found that increasing severity of sinonasal symptomatology is positively associated with the need for asthma-related oral corticosteroids in the past year. Moreover, we found that SNOT-22 score above a threshold of greater than 32 was quite sensitive (88.1\%), albeit with only a specificity of $41.2 \%$, in predicting the need for an asthma-related course of oral corticosteroids in the previous twelve-month period. Alternatively, a SNOT-22 score above a threshold of 65 was quite specific (91.2\%), albeit with a sensitivity of $38.1 \%$. We have previously found that the SNOT-22 score was associated with Asthma Control Test scores in asthmatic CRS patients ${ }^{(3)}$. Our results now show that the SNOT-22 score is also associated with the use of asthma-related oral corticosteroids in the past yearan indicator of past, present and future asthma outcomes-and the SNOT-22 score may have additional clinical utility as a test to identify asthmatic CRS patients who should be further queried about their asthma-related corticosteroid use. Our results show that the SNOT-22 score can be used as a versatile tool, based on the needs of the provider, with SNOT-22 scores in the "moderate" range as highly sensitive and SNOT-22 scores in the "severe" range as highly specific in detecting past asthma-related oral corticosteroid use ${ }^{(34)}$. In contrast, endoscopy score was not associated with participants who had needed asthma-related oral corticosteroid use in the past year and we did not find endoscopy score to be a good test for detection of past asthma-related oral corticosteroid use. That we did not find any relationship between endoscopy score and asthma-related oral corticosteroid use may be due to a weak association between these two variables - which is also consistent with the point estimate for the correlation of small effect size that we found - and our study was powered to detect associations of medium effect size. Our study results have important implications for patients with CRS and comorbid asthma. In addition to being a comorbid condition, CRS may also play a role in acute asthma exacerbations. It is unclear whether this interplay between CRS and asthma is related to concomitant and overlapping inflammatory pathways in the airway mucosa, as suggested by the unified airway hypothesis,(35) or whether it is related to discrete CRS symptomatology, which may directly aggravate the lower airway. Nevertheless and irrespective of mechanism, our results also immediately inform the clinical care of asthmatic CRS patients by providing 
a means for informing caretakers about the status of their CRS patients' asthma through the assessment of these patients' CRS sinonasal symptom severity.

The triggers for asthma exacerbations are numerous, and include upper respiratory viral infections, allergens, air pollutants, gastroesophageal reflux disease, obesity and psychological stress ${ }^{(11)}$. The common mechanism among these triggers is increased systemic inflammation leading to airway inflammation. CRS is a disease that is also characterized by sinonasal inflammation. Previous studies have shown that increased levels of inflammatory markers such as eosinophils and pro-inflammatory cytokines correlate with increasing CRS disease severity ${ }^{(36,37)}$. Increased CRS disease severity can lead to increased inflammation within the airway, which has the potential to trigger an asthma exacerbation. These previously described inflammatory and mechanistic findings parallel our findings that an increase in CRS sinonasal symptom severity may be associated with more frequent asthma exacerbations.

The findings of our study should be interpreted in the context of its limitations. Most pertinent, the number of asthma-related oral corticosteroids in the past year was based on participant reports at the time of enrollment and is subject to recall bias. Additionally, we did not distinguish oral corticosteroid courses by length or total dosage, which would have been informative. However, patient-reported medication use, reported in this manner, has been validated for clinical and research usage for many diseases including not only CRS ${ }^{(38)}$ but asthma as well ${ }^{(39)}$. Moreover, the National Heart, Lung and Blood Institute Expert Panel Report 3 recommends using patient-reported medication usage, including asthma-related systemic corticosteroid usage, independent of length or dosage, in the assessment of asthma control for titration of asthma treatment ${ }^{(11)}$. It is also important to consider that asthma may directly contribute to certain symptoms, such as cough, that are included in the SNOT-22. However, because the severity of nasal symptoms on the SNOT-
22 is associated with past asthma-related oral corticosteroid use with a magnitude similar to the severity of symptoms such as cough, which may have a contribution from asthma, it is likely that our findings do reflect associations with the severity of symptoms due to CRS. Additionally, we did not perform pulmonary function testing, which is a very cross-sectional measure in contrast to asthma-related systemic corticosteroid usage - our outcome measure of interest - which reflects past, present and future asthma characteristics. However, future studies of the association between CRS severity and asthma outcomes that incorporate objective metrics of pulmonary function would also be of interest.

CRS severity, as measured by the SNOT-22 score, is associated with the need for oral corticosteroid use for asthma in the past year, which supports the notion that upper and lower airway diseases may be interrelated. SNOT-22 can also be used as either a highly sensitive screening or highly specific tool to detect asthmatic CRS patients who have needed oral corticosteroids for asthma. Future studies should focus on evaluating whether metrics of CRS severity are predictive of asthma-related oral corticosteroid use in the future and whether treatment aimed at reducing CRS symptomatology decreases the frequency of asthma exacerbations.

\section{Acknowledgements}

No funding was used to support this study. The authors gratefully acknowledge the contribution of the patients who participated in this study.

\section{Authorship contribution}

KMP, LPH, RWB, APC, DSC, STG, ARS designed, performed the study and wrote/revised the manuscript.

\section{Conflict of interest}

The authors report no conflicts of interest.

\section{References}

1. Hoehle LP, Phillips KM, Bergmark RW Caradonna DS, Gray ST, Sedaghat AR. Symptoms of chronic rhinosinusitis differentially impact general health-related quality of life. Rhinology 2016 Sep 25;54(4):316322.

2. Phillips KM, Hoehle LP, Bergmark RW, Caradonna DS, Gray ST, Sedaghat AR. Acute Exacerbations Mediate Quality of Life Impairment in Chronic Rhinosinusitis. J Allergy Clin Immunol Pract 2016 Nov 2.

3. Phillips KM, Hoehle LP, Caradonna DS, Gray ST, Sedaghat AR. Association of severity of chronic rhinosinusitis with degree of comorbid asthma control. Ann Allergy
Asthma Immunol 2016 Dec;117(6):651-654.

4. Carey RM, Adappa ND, Palmer JN, Lee RJ, Cohen NA. Taste Receptors: Regulators of Sinonasal Innate Immunity. Laryngoscope Investig Otolaryngol 2016;1(4):88-95.

5. London NR, Lane AP. Innate Immunity and Chronic Rhinosinusitis: What We Have Learned From Animal Models. Laryngoscope Investig Otolaryngol 2016;1(3):49-56

6. Sedaghat AR, Phipatanakul W, Cunningham MJ. Atopy and the Development of Chronic Rhinosinusitis in Children with Allergic Rhinitis. J Allergy Clin Immunol Pract 2013;1 (6):689-691.

7. Sedaghat AR, Gray ST, Wilke CO, Caradonna
DS. Risk factors for development of chronic rhinosinusitis in patients with allergic rhinitis. Int Forum Allergy Rhinol 2012 SepOct;2(5):370-375.

8. Van Crombruggen K, Zhang N, Gevaert P, Tomassen P, Bachert C. Pathogenesis of chronic rhinosinusitis: inflammation. J Allergy Clin Immunol 2011 Oct;128(4):728732.

9. Rosenfeld RM, Piccirillo JF, Chandrasekhar SS, Brook I, Ashok Kumar K, Kramper M, et al. Clinical practice guideline (update): adult sinusitis. Otolaryngol Head Neck Surg 2015 Apr;152(2 Suppl):S1-S39.

10. Fokkens WJ, Lund VJ, Mullol J, Bachert C, Alobid I, Baroody F, et al. European Position 
Paper on Rhinosinusitis and Nasal Polyps 2012. Rhinol Suppl 2012 Mar;(23)(23):3 p preceding table of contents, 1-298.

11. EPR-3. NAEPP expert panel report 3: guidelines for the diagnosis and treatment of asthma. Bethesda (MD): US Department of Health and Human Services; National Institutes of Health; National Heart, Lung, and Blood Institute, 2007.

12. Jarvis D, Newson R, Lotvall J, Hastan D, Tomassen P, Keil T, et al. Asthma in adults and its association with chronic rhinosinusitis: the GA2LEN survey in Europe. Allergy 2012 Jan;67(1):91-98.

13. Dixon AE. Rhinosinusitis and asthma: the missing link. Curr Opin Pulm Med 2009 Jan;15(1):19-24

14. Seybt MW, McMains KC, Kountakis SE. The prevalence and effect of asthma on adults with chronic rhinosinusitis. Ear Nose Throat J 2007 Jul;86(7):409-411.

15. Slavin RG. The upper and lower airways: the epidemiological and pathophysiologica connection. Allergy Asthma Proc 2008 NovDec;29(6):553-556.

16. Annesi-Maesano I. Epidemiological evidence of the occurrence of rhinitis and sinusitis in asthmatics. Allergy 1999;54 Suppl 57:7-13.

17. Dennis SK, Lam K, Luong A. A Review of Classification Schemes for Chronic Rhinosinusitis with Nasal Polyposis Endotypes. Laryngoscope Investig Otolaryngol 2016 Oct;1(5):130-134.

18. Promsopa C, Kansara S, Citardi MJ, Fakhri S, Porter P, Luong A. Prevalence of confirmed asthma varies in chronic rhinosinusitis subtypes. Int Forum Allergy Rhinol 2016 Apr;6(4):373-377.

19. Halawi AM, Smith SS, Chandra RK Chronic rhinosinusitis: epidemiology and cost. Allergy Asthma Proc 2013 JulAug;34(4):328-334.

20. Pearlman AN, Chandra RK, Chang D, Conley DB, Tripathi-Peters A, Grammer LC, et al. Relationships between severity of chronic rhinosinusitis and nasal polyposis, asthma, and atopy. Am J Rhinol Allergy 2009 MarApr;23(2):145-148.

21. Lin DC, Chandra RK, Tan BK, Zirkle W, Conley DB, Grammer LC, et al. Association between severity of asthma and degree of chronic rhinosinusitis. Am J Rhinol Allergy 2011 JulAug;25(4):205-208.

22. ten Brinke $A$, Sterk PJ, Masclee AA, Spinhoven $\mathrm{P}$, Schmidt JT, Zwinderman AH, et al. Risk factors of frequent exacerbations in difficult-to-treat asthma. Eur Respir J 2005 Nov;26(5):812-818.

23. Randhawa I, Klaustermeyer WB. Oral corticosteroid-dependent asthma: a 30-year review. Ann Allergy Asthma Immunol 2007 Oct;99(4):291-302; quiz 302-3, 370.

24. Kroegel C. Global Initiative for Asthma (GINA) guidelines: 15 years of application. Expert Rev Clin Immunol 2009 May;5(3):239-249.

25. Hopkins C, Gillett S, Slack R, Lund VJ, Browne JP. Psychometric validity of the 22-item Sinonasal Outcome Test. Clin Otolaryngol 2009 Oct;34(5):447-454

26. Lund VJ, Kennedy DW. Staging for rhinosinusitus. Otolaryngol Head Neck Surg 1997 Sep;117(3 Pt 2):S35-40.

27. Robin $X$, Turck N, Hainard A, Tiberti N, Lisacek F, Sanchez JC, et al. pROC: an opensource package for $\mathrm{R}$ and $\mathrm{S}+$ to analyze and compare ROC curves. BMC Bioinformatics 2011 Mar 17:12:77-2105-12-77.

28. Batra PS, Tong L, Citardi MJ. Analysis of comorbidities and objective parameters in refractory chronic rhinosinusitis. Laryngoscope 2013 Dec;123 Suppl 7:S1-11.

29. Ek A, Middelveld RJ, Bertilsson H, Bjerg A, Ekerljung L, Malinovschi A, et al. Chronic rhinosinusitis in asthma is a negative predictor of quality of life: results from the Swedish GA(2)LEN survey. Allergy 2013 Oct;68(10):1314-1321.

30. Bresciani M, Paradis L, Des Roches A, Vernhet H, Vachier I, Godard P, et al. Rhinosinusitis in severe asthma. J Allergy Clin Immunol 2001 Jan;107(1):73-80.

31. Sumino K, O'Brian K, Bartle B, Au DH, Castro $M$, Lee TA. Coexisting chronic conditions associated with mortality and morbidity in adult patients with asthma. J Asthma 2014 Apr;51(3):306-314.

32. Braman SS. The global burden of asthma. Chest 2006 Jul;130(1 Suppl):4S-12S.

33. Moore WC, Bleecker ER, Curran-Everett $D$, Erzurum SC, Ameredes BT, Bacharier L, et al. Characterization of the severe asthma phenotype by the National Heart, Lung, and
Blood Institute's Severe Asthma Research Program. J Allergy Clin Immunol 2007 Feb;119(2):405-413.

34. Toma S, Hopkins C. Stratification of SNOT-22 scores into mild, moderate or severe and relationship with other subjective instruments. Rhinology 2016 Jun;54(2):129-133.

35. Feng $\mathrm{CH}$, Miller MD, Simon RA. The united allergic airway: connections between allergic rhinitis, asthma, and chronic sinusitis. Am J Rhinol Allergy 2012 MayJun;26(3):187-190

36. Szucs E, Ravandi S, Goossens A, Beel M, Clement PA. Eosinophilia in the ethmoid mucosa and its relationship to the severity of inflammation in chronic rhinosinusitis. Am J Rhinol 2002 May-Jun;16(3):131-134.

37. Min HJ, Kim SJ, Kim TH, Chung HJ, Yoon $\mathrm{JH}$, Kim CH. Level of secreted HMGB1 correlates with severity of inflammation in chronic rhinosinusitis. Laryngoscope 2015 Jul;125(7):E225-30.

38. Gliklich RE, Metson R. Techniques for outcomes research in chronic sinusitis. Laryngoscope 2015 Oct;125(10):2238-2241.

39. Nathan RA, Sorkness CA, Kosinski M, Schatz M, Li JT, Marcus P, et al. Development of the asthma control test: a survey for assessing asthma control. J Allergy Clin Immunol 2004 Jan;113(1):59-65.

\author{
Ahmad R. Sedaghat, MD, PhD \\ Department of Otolaryngology \\ Massachusetts Eye and Ear Infirmary \\ 243 Charles Street \\ Boston, MA, \\ USA 02114
}

Tel: +001 617-573-6011

Fax: +001 617-573-6845

E-mail:

ahmad_sedaghat@meei.harvard.edu 